\title{
Examining the Necessity of Attention for Consciousness in Iconic Memory Using Modified Stroop Paradigm
}

\section{Mehdi Afzalinia, Imanollah Bigdeli*, Javad Salehi Fadardi}

Department of Psychology, Faculty of Educational Sciences and Psychology, Ferdowsi University of Mashhad, Mashhad, Iran

\section{ABSTRACT}

Introduction: One of the main challenging issues in cognitive science is the role of attention in consciousness. It is generally believed that attention is essential for consciousness. However, some studies have shown in some conditions, such as iconic memory, consciousness happens without attention. In previous studies, using iconic memory dual tasks in which attention is locally diverted to peripheral task in order to examine consciousness in the central task has caused the problem of expectation and previous knowledge in perception of the stimuli. The purpose of the present study was to test attention necessity for consciousness in iconic memory using the modified Stroop paradigm. Materials and Methods: Thirty Participants subjects were selected and randomly assigned to the partial and whole report groups. Results: Participants had significantly more correct responses in the partial group compared to the whole report group both in congruent and incongruent conditions. This indicates that attentional bias to word processing in incongruent conditions did not affect the correct responses compared to congruent conditions. Conclusion: Attention is not necessary for consciousness in iconic memory.

*Corresponding Author: Imanollah Bigdeli

Email: ibigdeli@um.ac.ir 
بررسى استلزام توجه براى آتاهى در حافظهُ حسى با استفاده از پارادايم تعديل يافتـُ استروٍ

مهدى افضلى نيا، ايمان الله بيگدلى"، جواد صالحى فدردى

كروه روانشناسى، دانشكده علوم تربيتى و روانشناسى ، دانشحاه فردوسى مشهد، مشهد، ايران

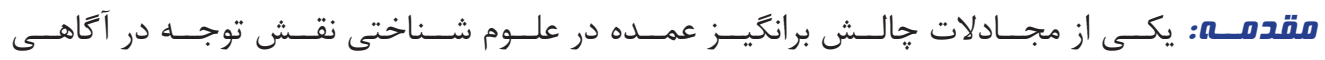

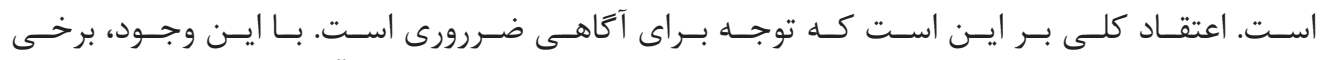

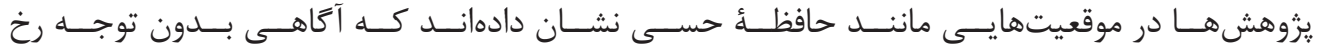

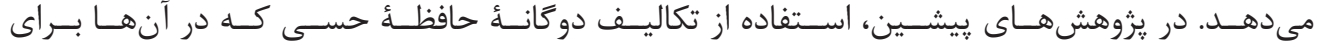

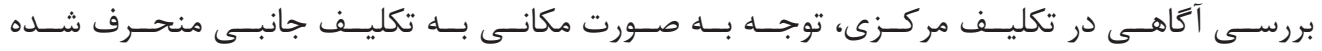

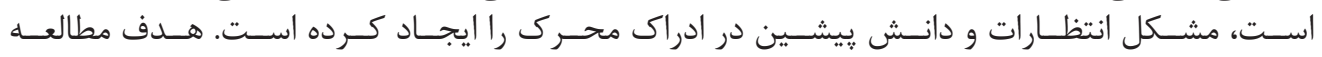

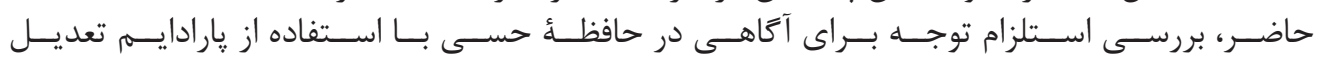

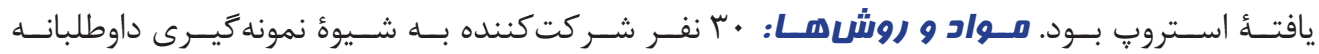

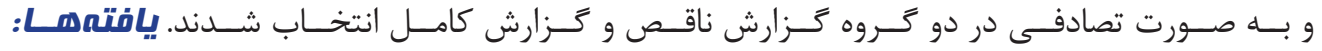

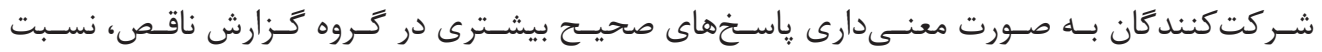

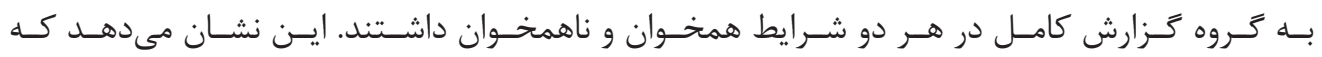

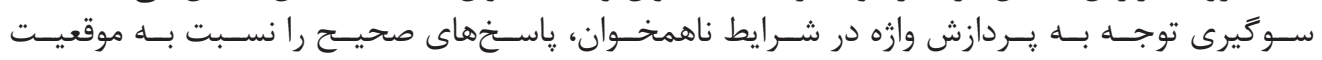

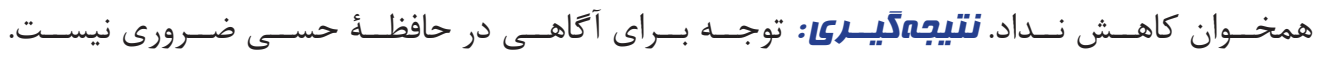




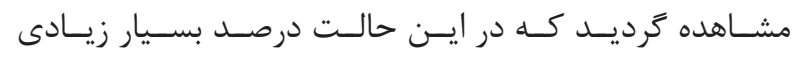

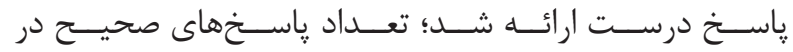

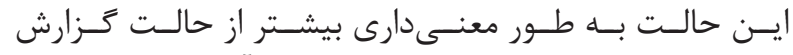

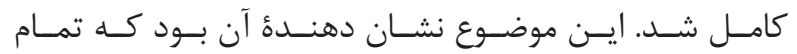

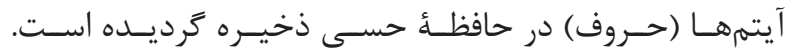

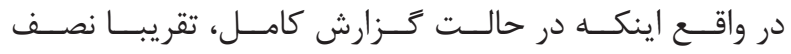

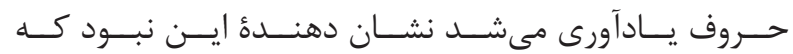

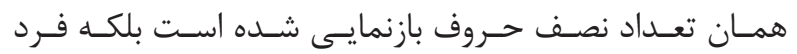

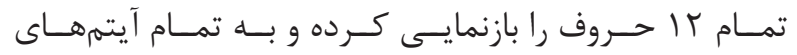

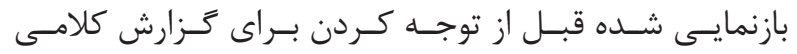

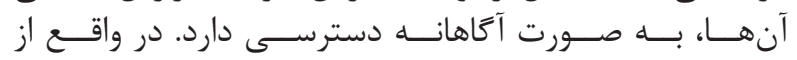

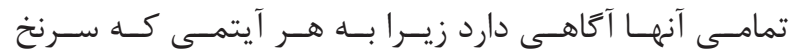

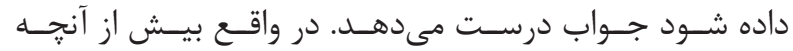

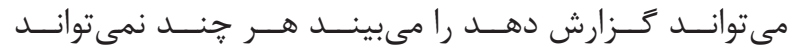

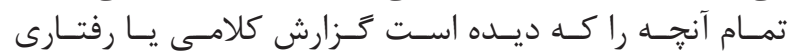

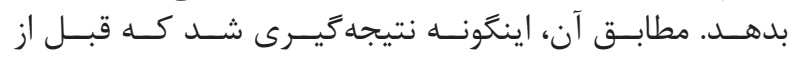

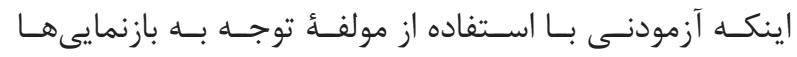

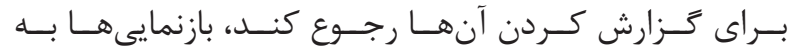

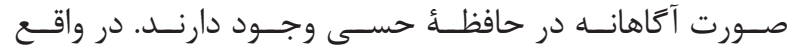

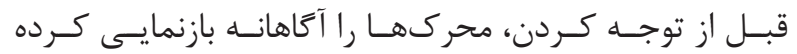

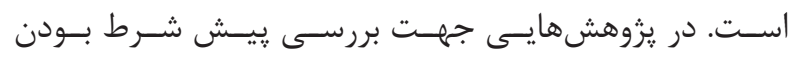

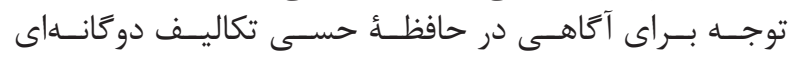

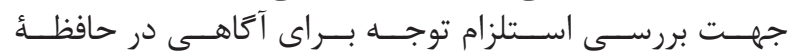

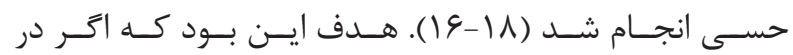

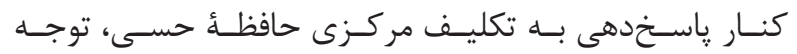

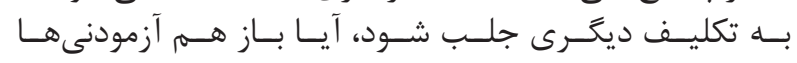

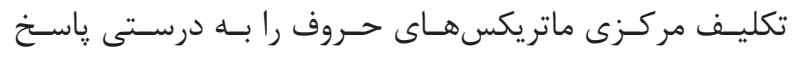

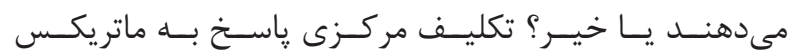

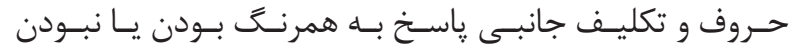

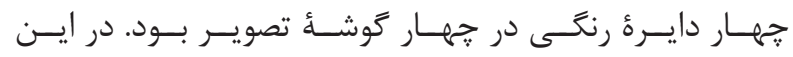

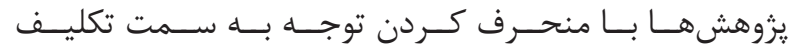

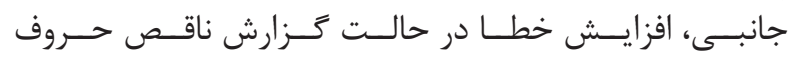

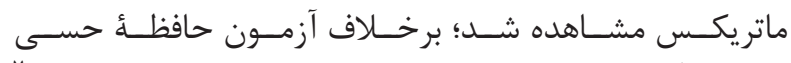

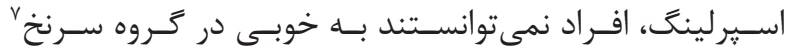

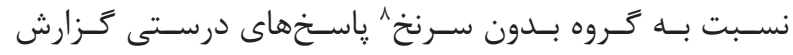

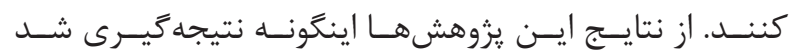

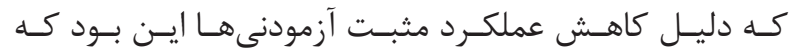

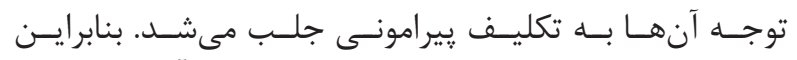

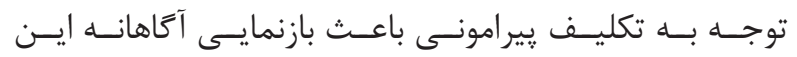

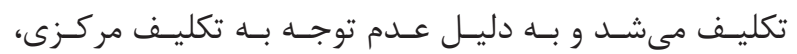

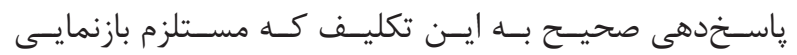

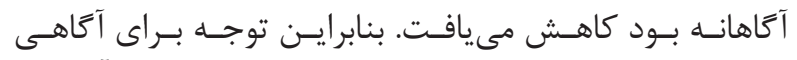

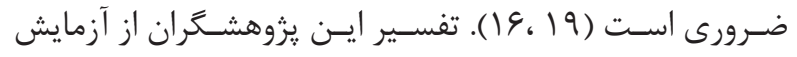

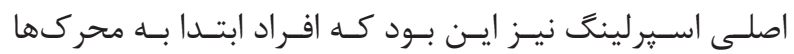

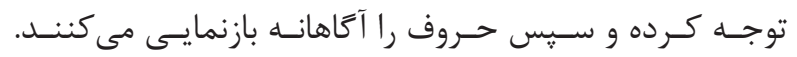

${ }^{1}$ Attention

${ }^{2}$ Consciousness

${ }^{3}$ Iconic memory

${ }^{4}$ Sperling
مقدمه

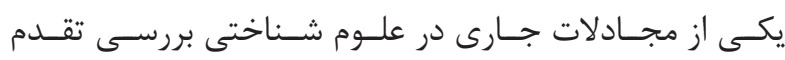

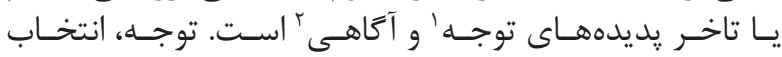

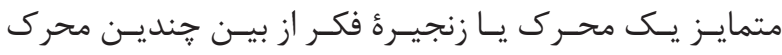

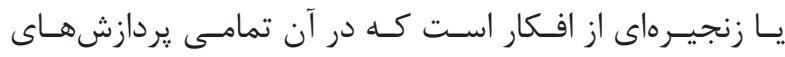

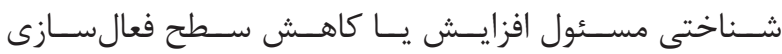

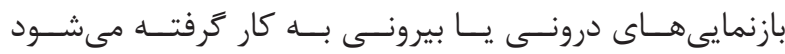

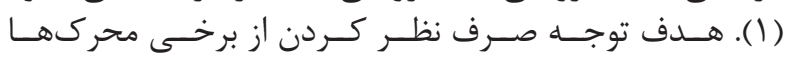

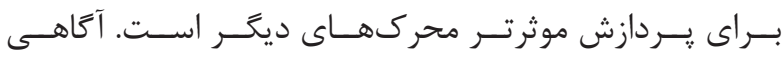

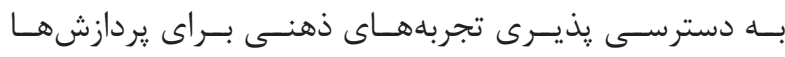

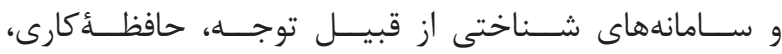

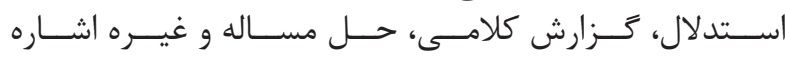

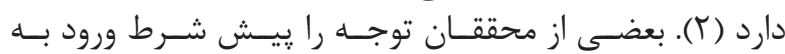

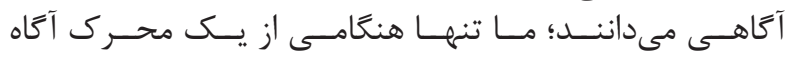

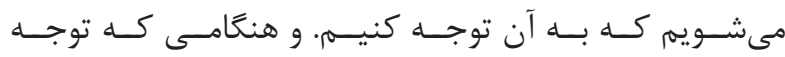

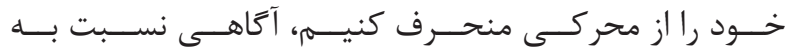

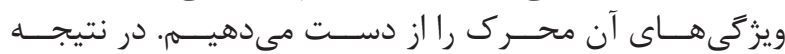

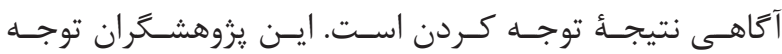

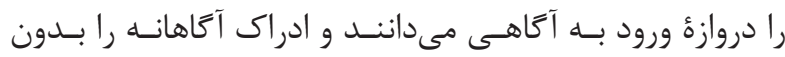

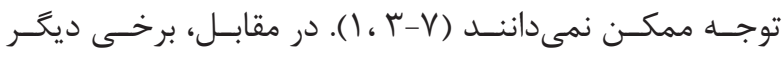

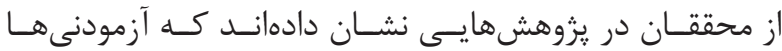

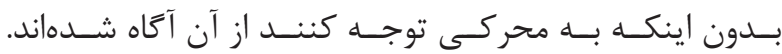

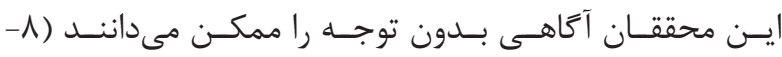

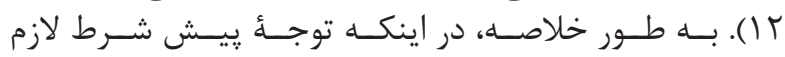

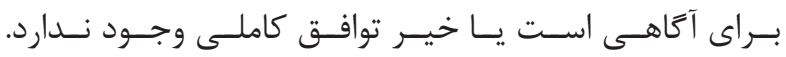

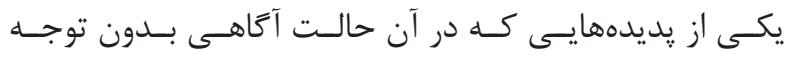

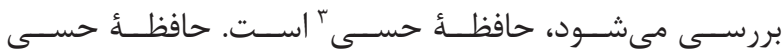

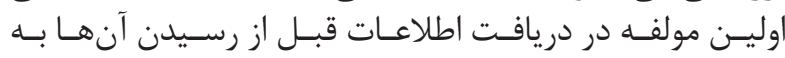

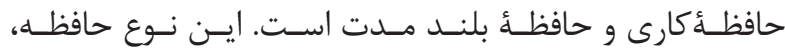

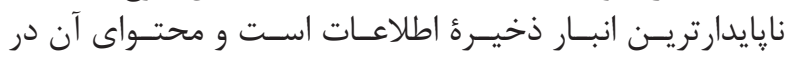

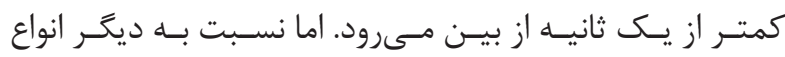

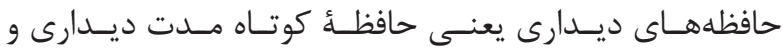

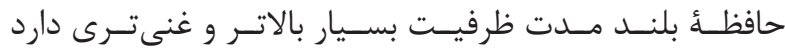

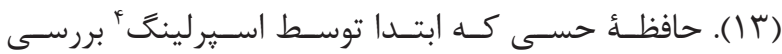

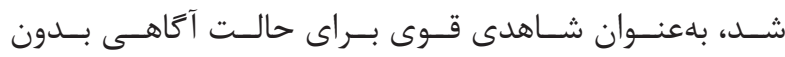

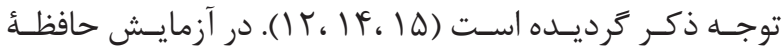

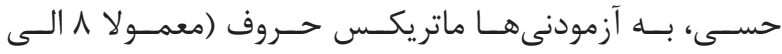

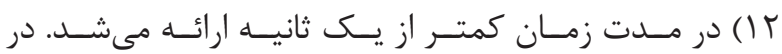

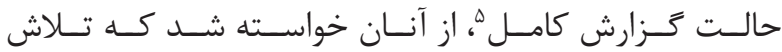

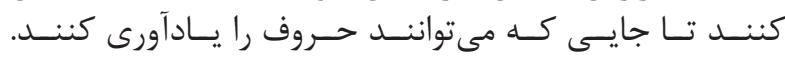

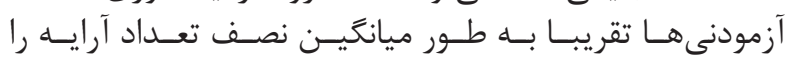

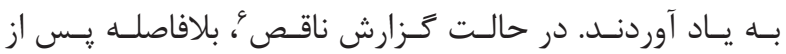

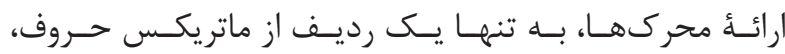

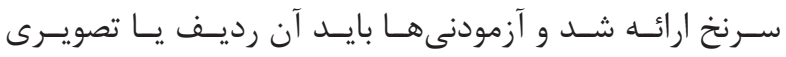

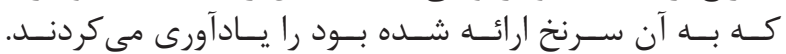

\footnotetext{
${ }^{5}$ Whole report

${ }^{6}$ Partial report

${ }^{7}$ Cue group

${ }^{8}$ No-cue group
} 


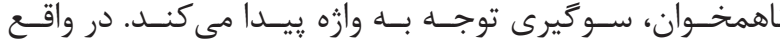

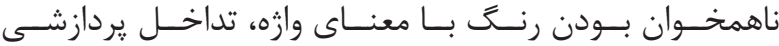

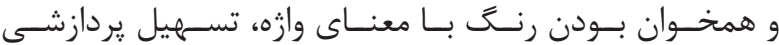

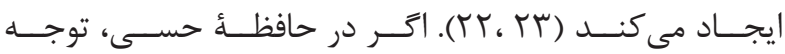

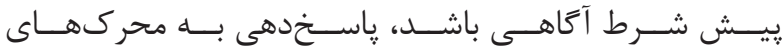

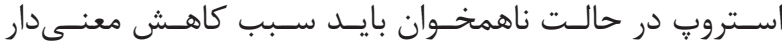

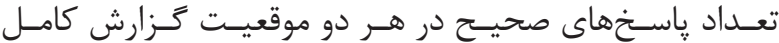

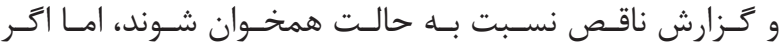

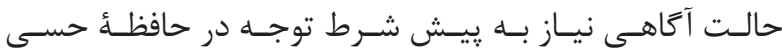

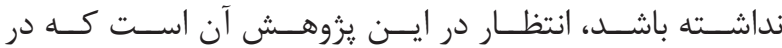

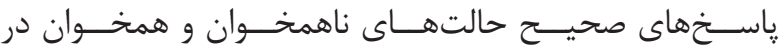

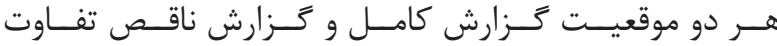

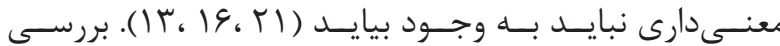

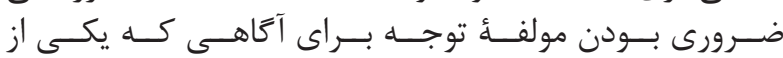

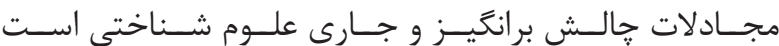

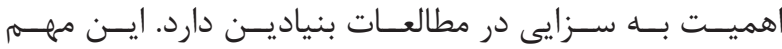

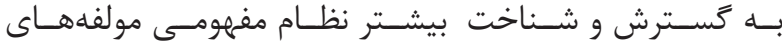

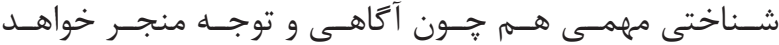

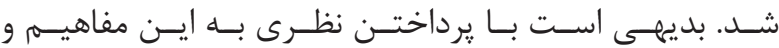

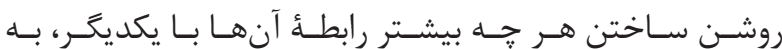

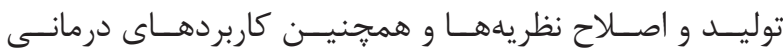

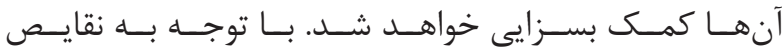

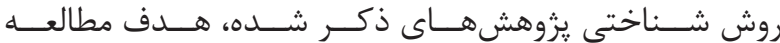

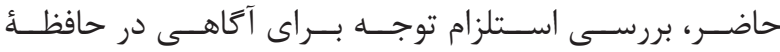

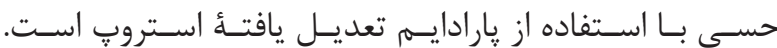

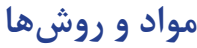

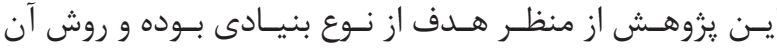

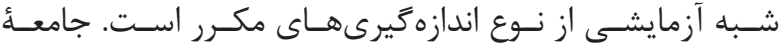

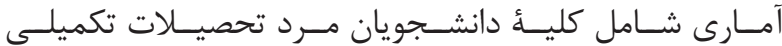

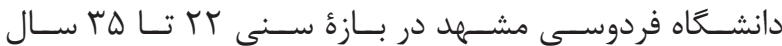

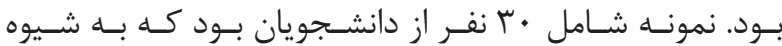

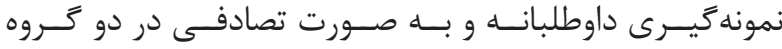

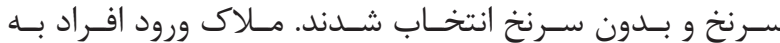

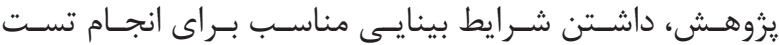

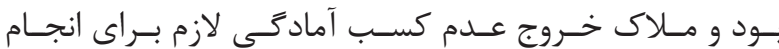

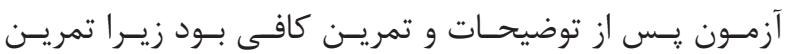

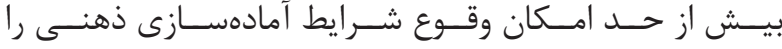

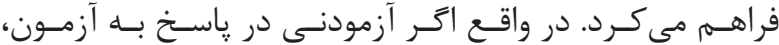

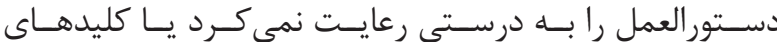

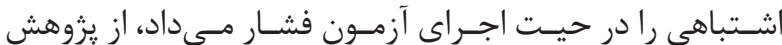

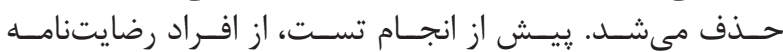

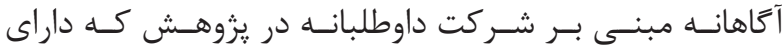

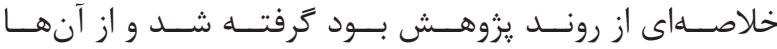

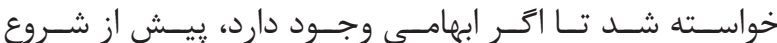

${ }^{9}$ Unconscious

${ }^{10}$ Prior knowledge

${ }^{11}$ Expectations

${ }^{12}$ Property

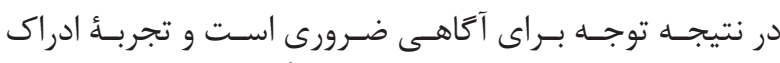

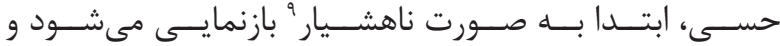

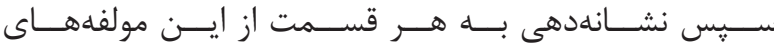

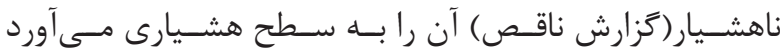

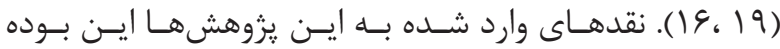

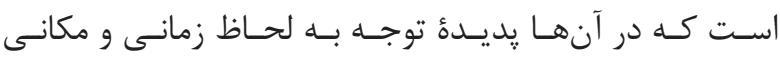

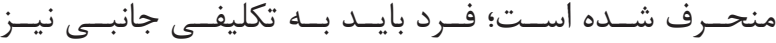

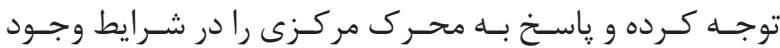

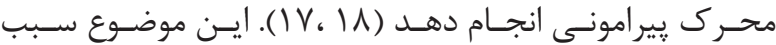

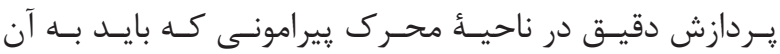

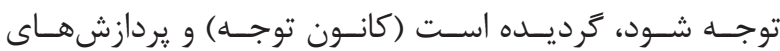

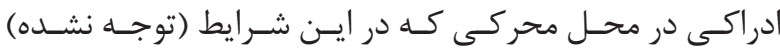

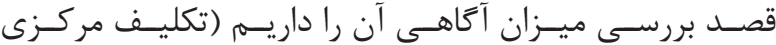

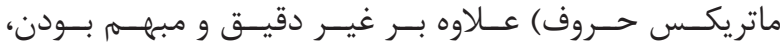

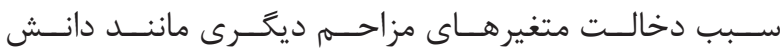

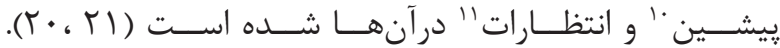

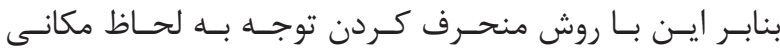

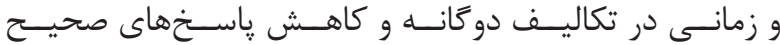

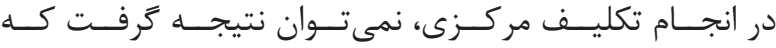

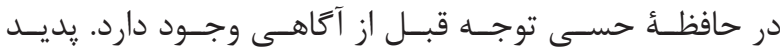

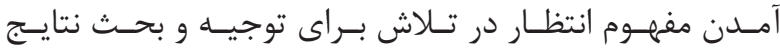

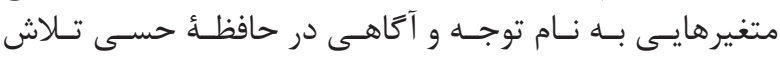

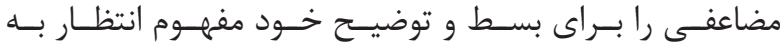

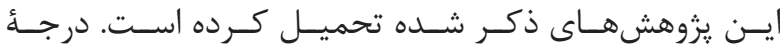

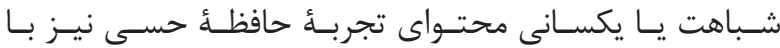

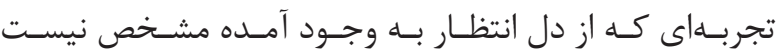

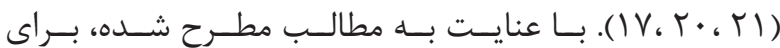

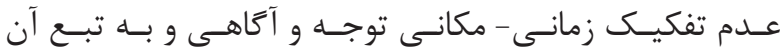

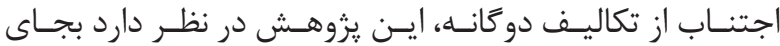

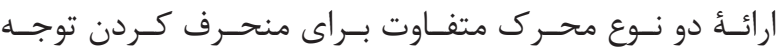

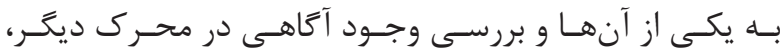

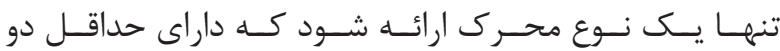

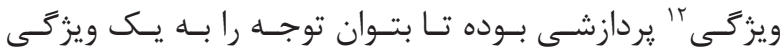

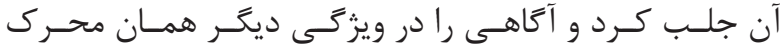

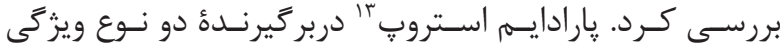

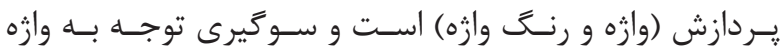

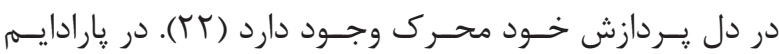

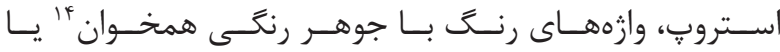

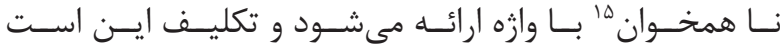

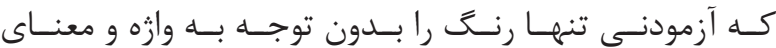

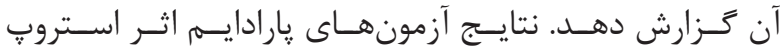

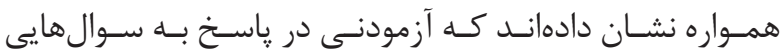

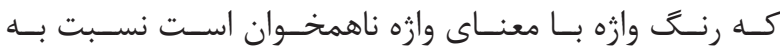

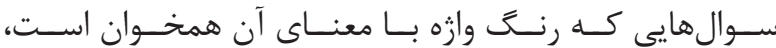

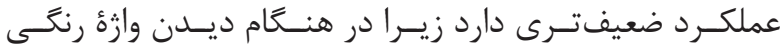

\footnotetext{
${ }^{13}$ Stroop paradigm

${ }^{14}$ Congruent

${ }^{15}$ Incongruent
} 


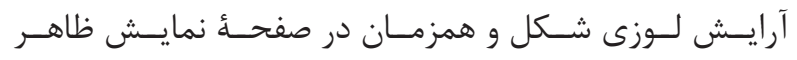

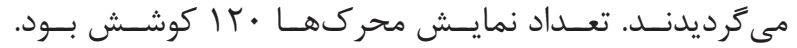

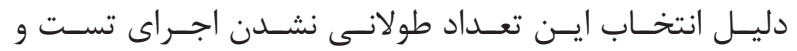

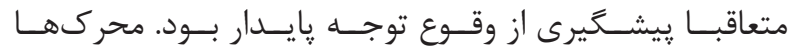

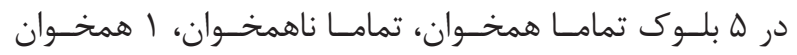

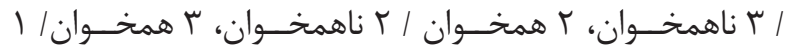

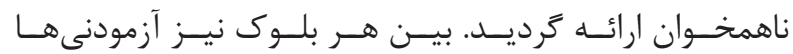

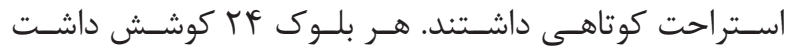

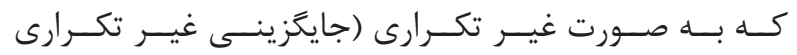

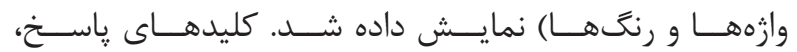

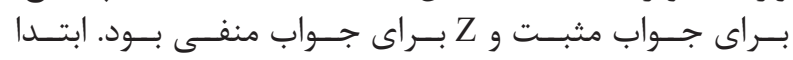

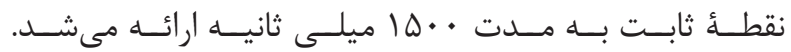

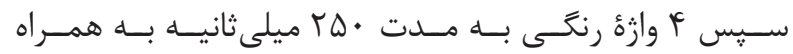

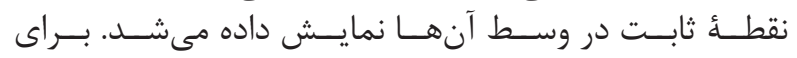

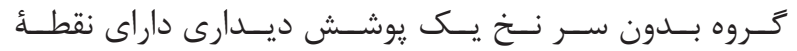

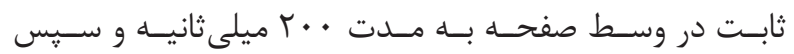

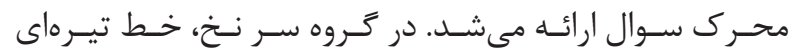

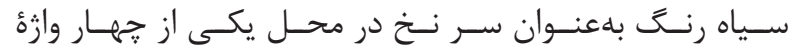

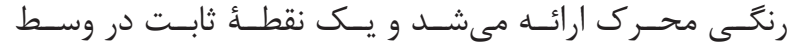

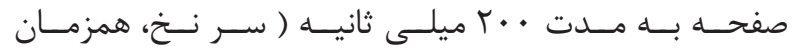

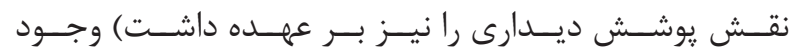

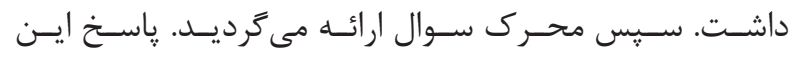

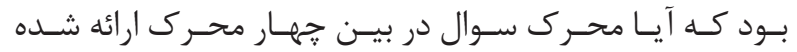

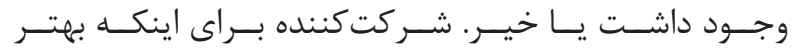

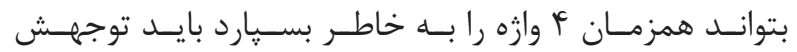

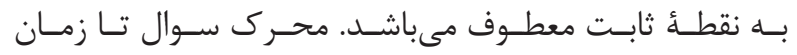

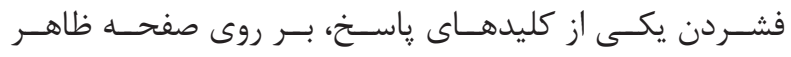

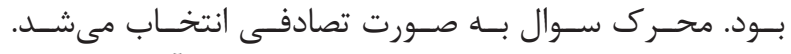

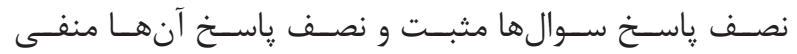

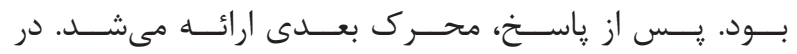

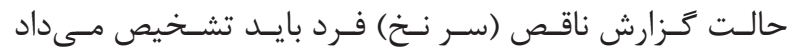

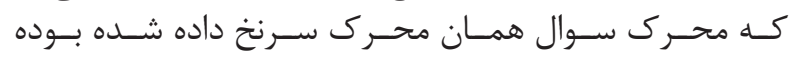

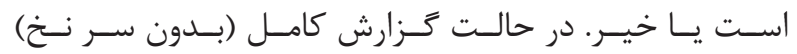

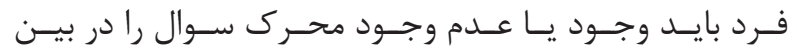

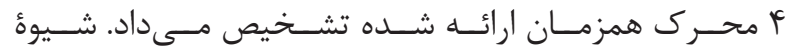

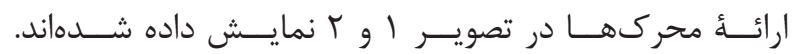

\section{روش تجزيه و تحليل دادهها}

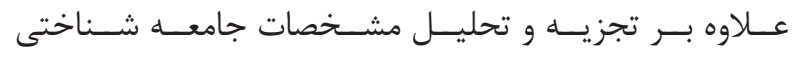

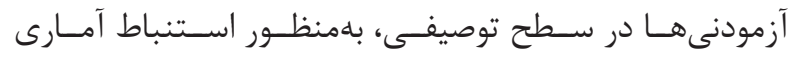

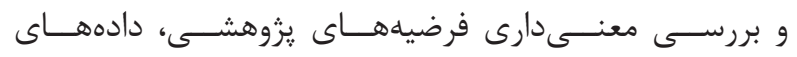

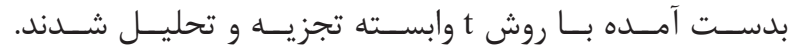

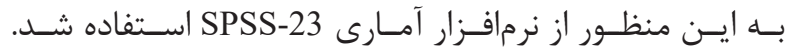

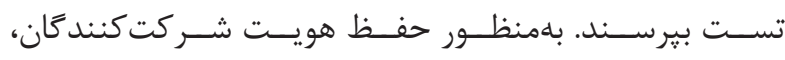

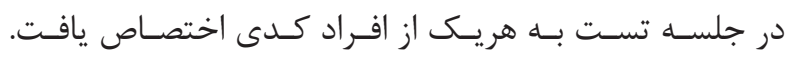

\section{آززار يزوهش استت تعدو تعديل يافته}

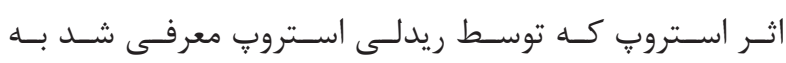

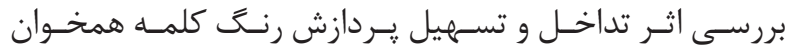

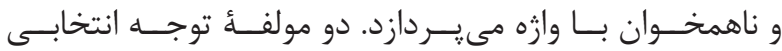

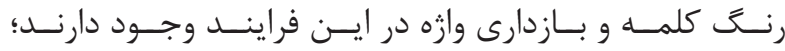

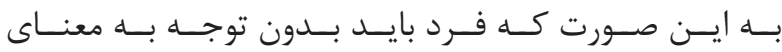

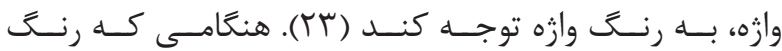

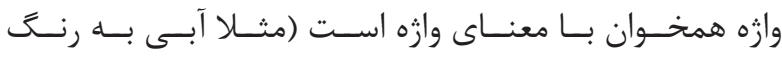

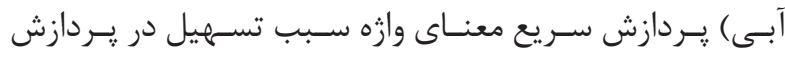

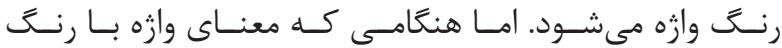

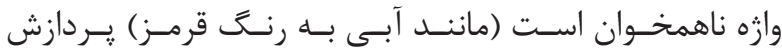

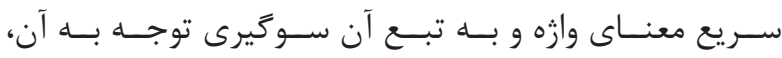

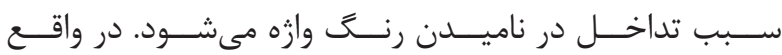

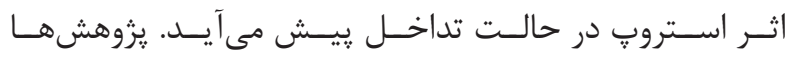

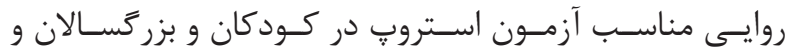

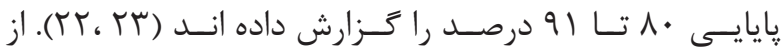

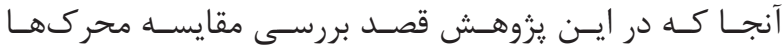

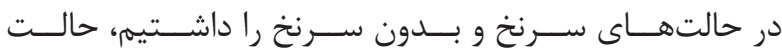

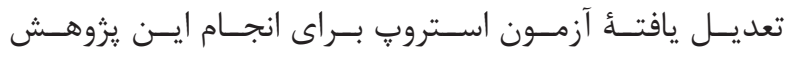

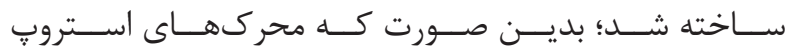

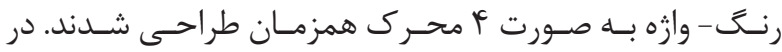

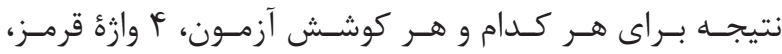

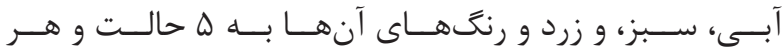

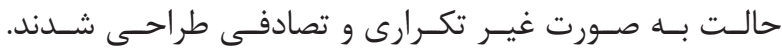

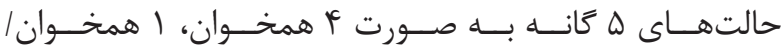

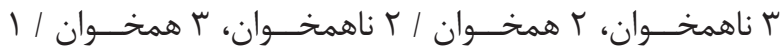

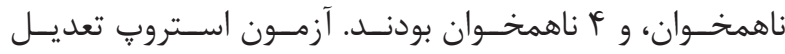

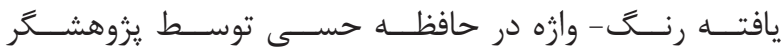

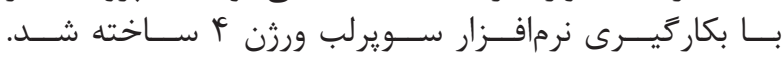

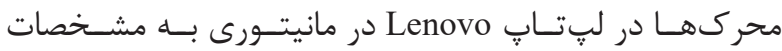

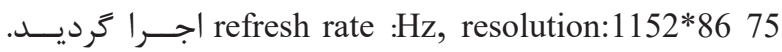

\section{روش اجراى يخوهش}

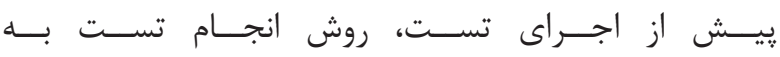

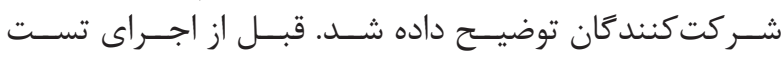

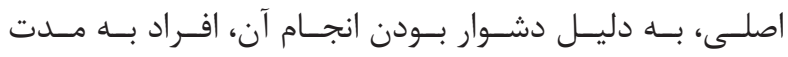

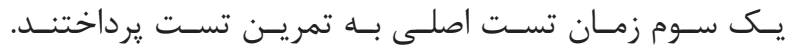

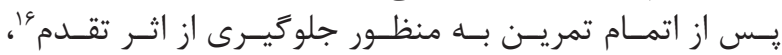

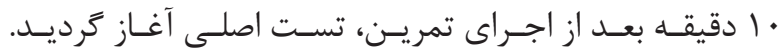

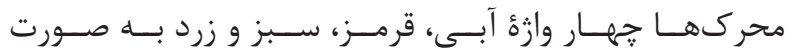




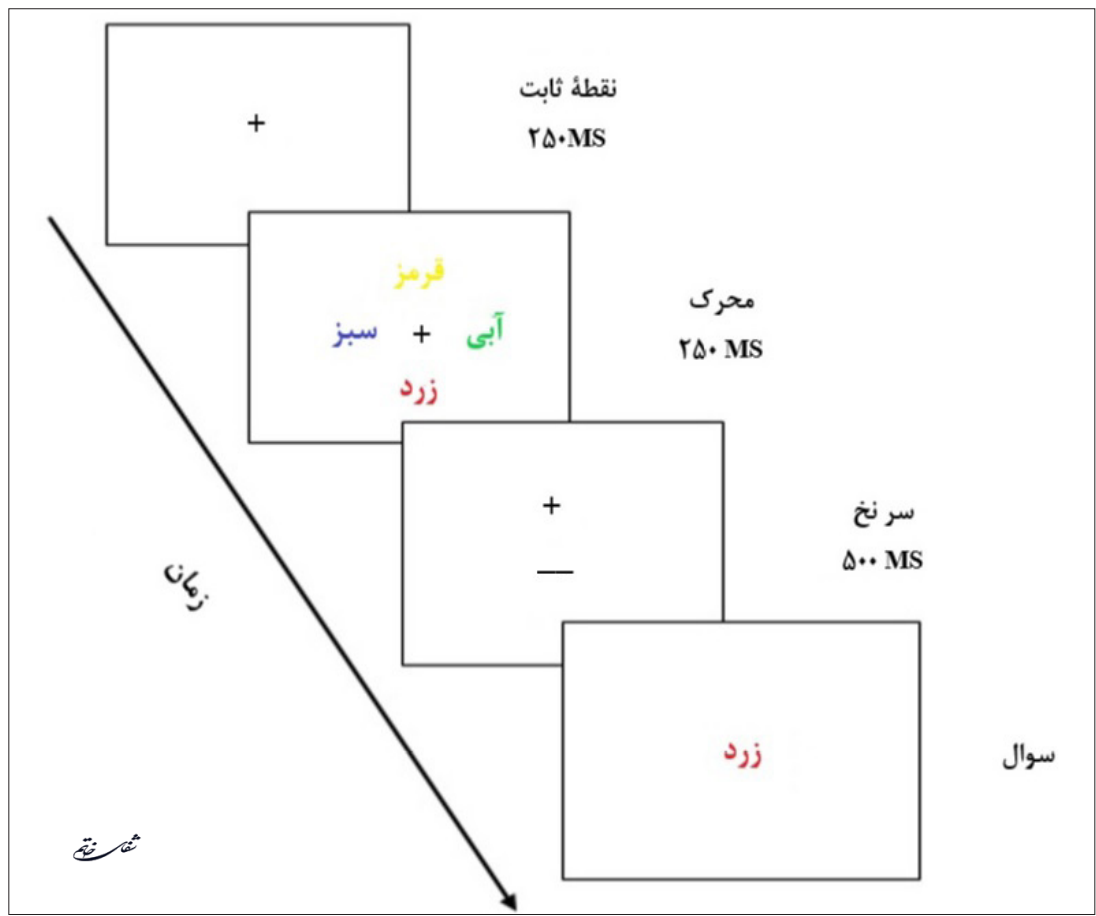

تصوير ا- شيوء ارائهُ محرك در كروه سر نخ

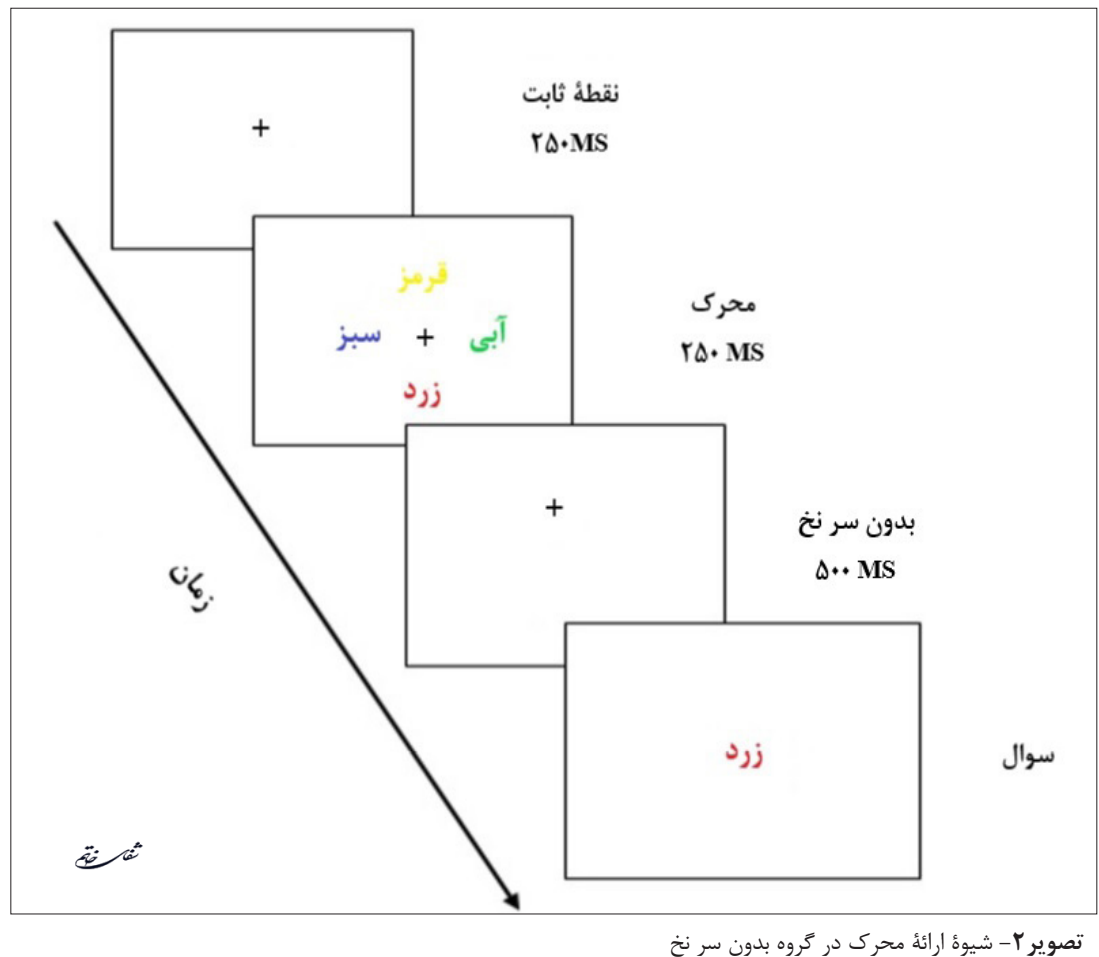

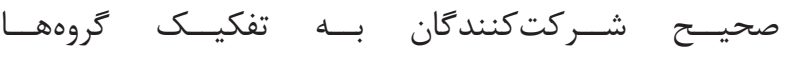

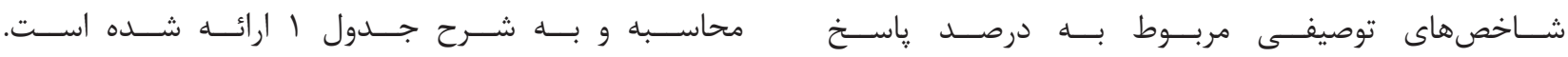

جدول ا- برخى شاخصهاى توصيفى متغير درصد پاسخ صحيح به تفكيك گروهها

\begin{tabular}{|c|c|c|c|c|}
\hline انحراف معيار & ميانكين (انحراف از ميانكين) & تعداد & مراحل & متغير \\
\hline $9 / \cdot 9$ & $\Delta 9 / \varphi \wedge( \pm \cdot \mid \Lambda F)$ & 10 & بدون سر نخ & \multirow{2}{*}{ درصد باسخ صحيح } \\
\hline T/YF & $V \omega / \Lambda r( \pm r / r \Delta)$ & 10 & با سر نخ & \\
\hline
\end{tabular}




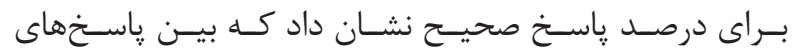

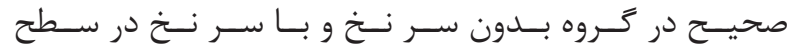

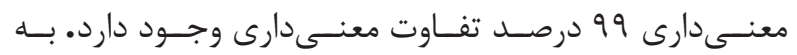

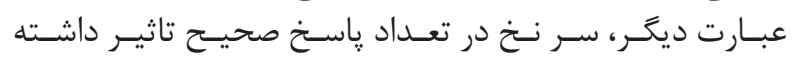

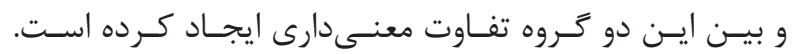

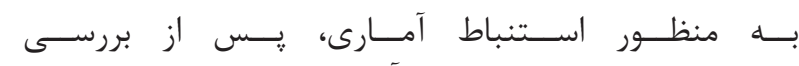

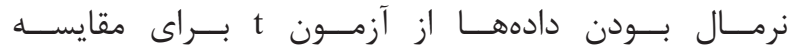

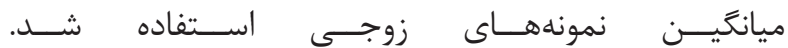

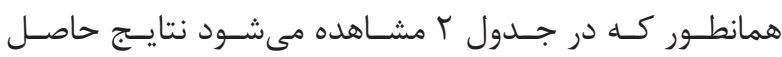

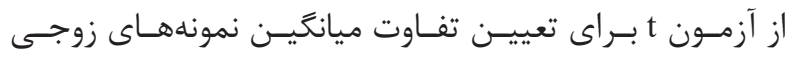

جدول r- مقايسٔ بين پاسخهاى صحيح در حالت سر نخدهى نسبت به حالت بدون سر نخ

\begin{tabular}{|c|c|c|c|c|}
\hline $\mathbf{t}$ & سطح معنى دارى & درجهُ آزادى & \multicolumn{2}{|c|}{ متغير } \\
\hline \multirow{2}{*}{ 9/V99 } & \multirow{2}{*}{$\cdot 1 \cdots$} & \multirow{2}{*}{ If } & بدون سر نخ & درصد پاسخ \\
\hline & & & با سر نخ & صحيح \\
\hline
\end{tabular}

جدول بـ مقايسٔ بين ياسخهاى صحيح در حالت سر نخدهى نسبت به حالت بدون سر نخ به تفكيك حالات

\begin{tabular}{|c|c|c|c|c|}
\hline$t$ & $\mathbf{P}$ & درجة آزادى & \multicolumn{2}{|c|}{ متثير } \\
\hline$r / \mu+\mu$ & 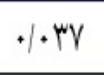 & If & F أهمخوان/با سر نيخ & fl همخروان/بدون سر نيخ \\
\hline$r / V \Delta \cdot$ & $H \cdot \cdot r$ & If & " همخوان، ا ناميدخوان/ با سر نغ & " هميخوان، ا ناهمخوان/ بدون سر نيخ \\
\hline $1 / \Delta f$. & $. / 1 \leqslant 4$ & If & r همخروان، ب ناميخولان/با سر نيخ & r همختوان، Y ناهمخوان/ بدون سر نخ \\
\hline r/GYA & $+\cdots$ & If & 1 همخوان، ب ناهمخوان/ با سر نيخ & ا همخوان، ب ناهمخوان/بدون سر نخ \\
\hline 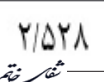 & $H \cdot Y F$ & If & f أناهيخوان/ با سر نيخ & fا نامهيخوان/ بدون سر نيخ \\
\hline
\end{tabular}

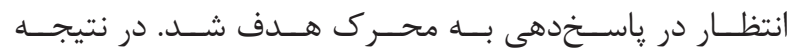

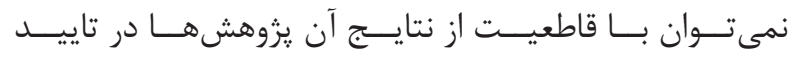

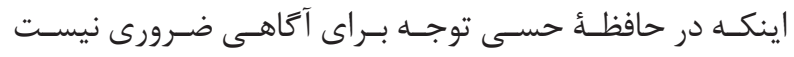

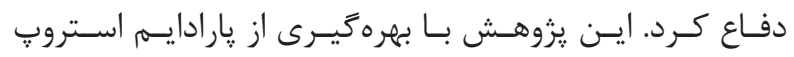

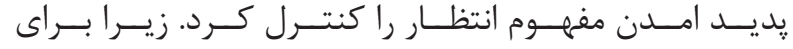

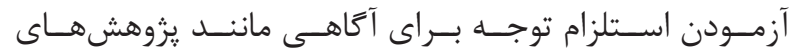

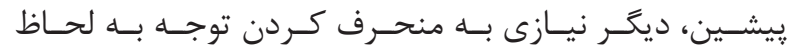

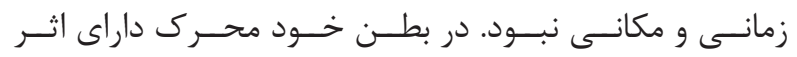

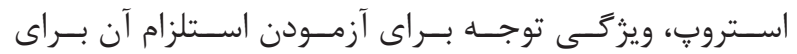

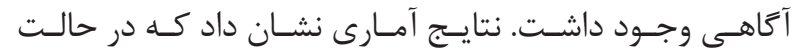

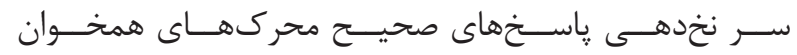

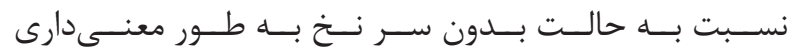

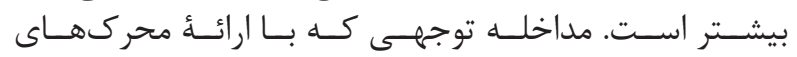

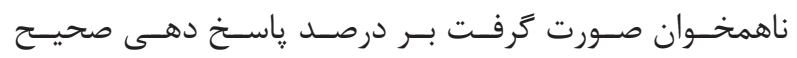

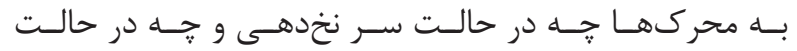

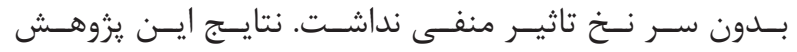

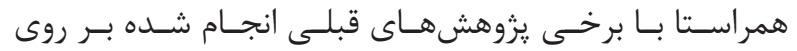

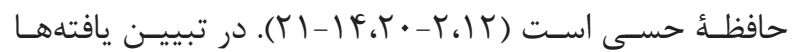

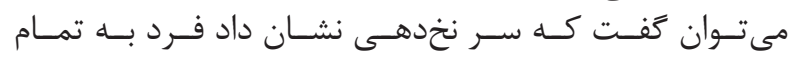

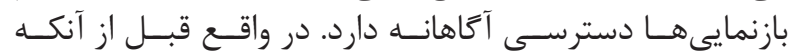

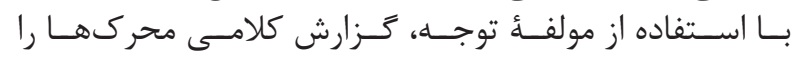

نتايـج حاصـل از آزمــون t در جــدول ب بــراى تعييـن تفــاوت

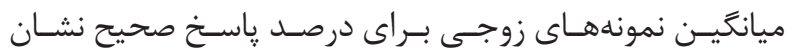

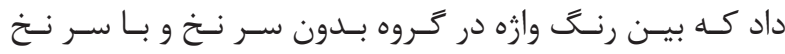

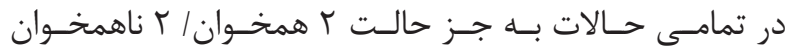

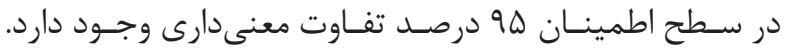
بحث و نتيجه كيرى

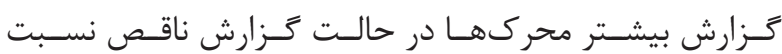

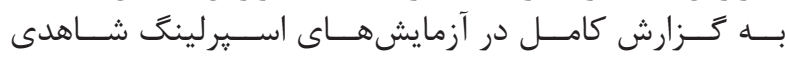

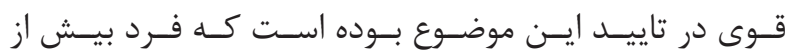

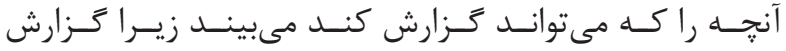

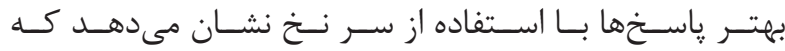

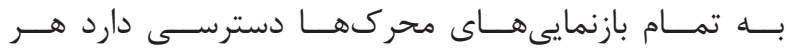

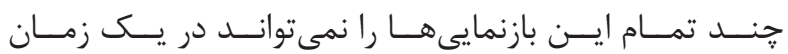

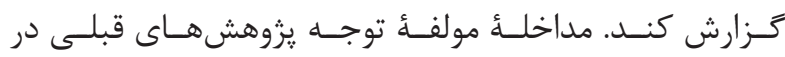

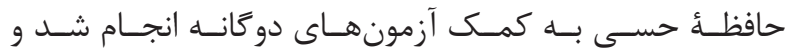

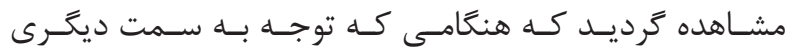

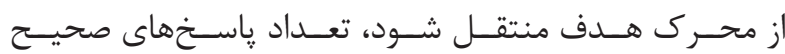

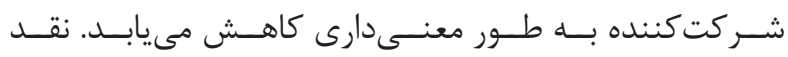

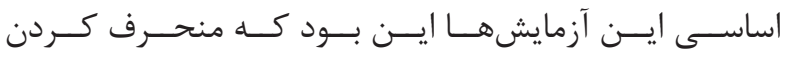

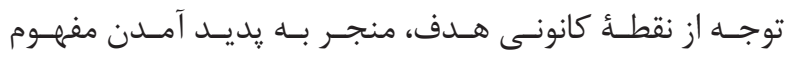




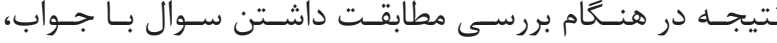

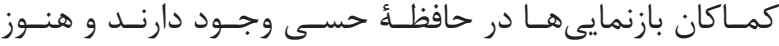

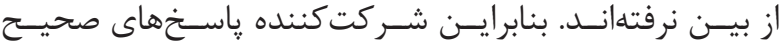

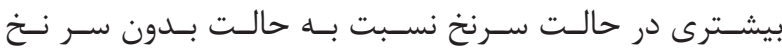

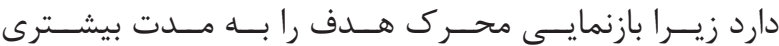

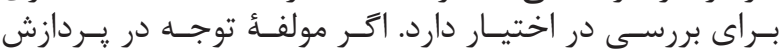

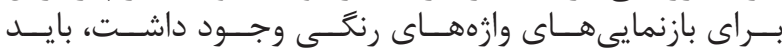

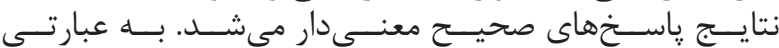

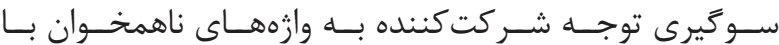

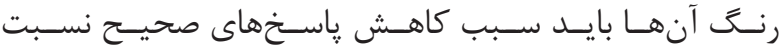

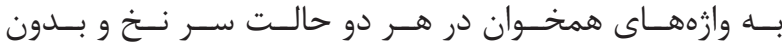

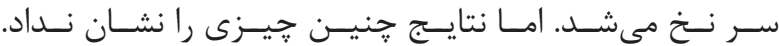

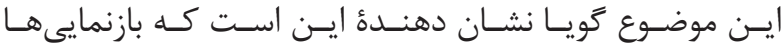

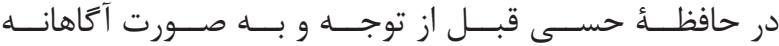

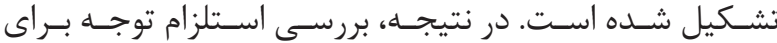

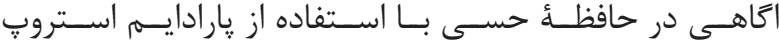

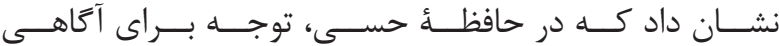

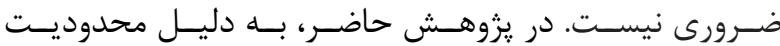

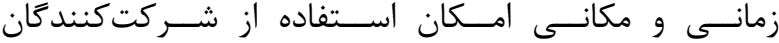

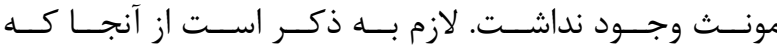

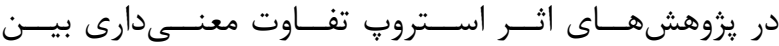

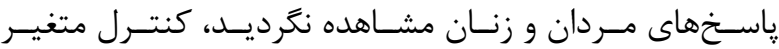

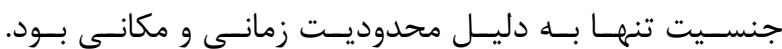

1. Posner M. Attentional networks and consciousness. Frontiers in psychology. 2012; 3: 64.

2. Block N. Consciousness, accessibility, and the mesh between psychology and neuroscience. Behavioral and brain sciences. 2007; 30(5-6): 481-99.

3. O'Regan JK, Noë A. A sensorimotor account of vision and visual consciousness. Behavioral and brain sciences. 2001; 24(5): 939-73. 4. Jackendoff R. How language helps us think. Pragmatics \& Cognition. 1996; 4(1): 1-34.

5. Marchetti G. Against the view that consciousness and attention are fully dissociable. Frontiers in psychology. 2012; 3: 36.

6. Cohen MA, Dennett DC. Consciousness cannot be separated from function. Trends in cognitive sciences. 2011; 15(8): 358-64.

7. Mack A, Rock I. Inattentional blindness (Vol. 33). Cambridge, MA: MITpress. 1998.

8. Koch C, Tsuchiya N. Attention and consciousness: two distinct brain processes. Trends Cogn Sci. 2007; 11(1): 16-22.

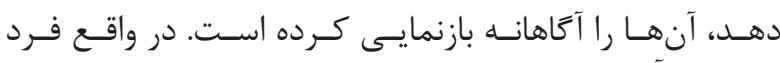

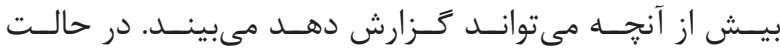

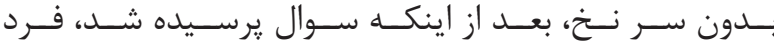

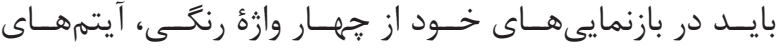

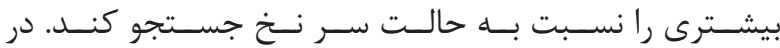

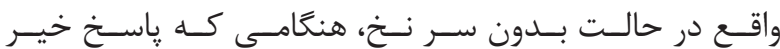

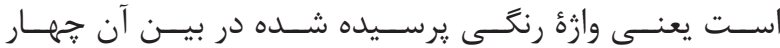

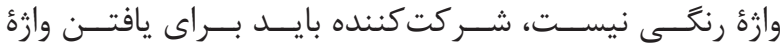

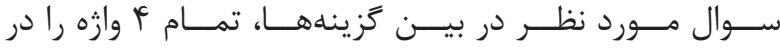

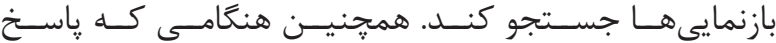

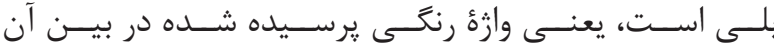

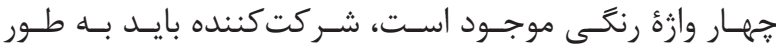

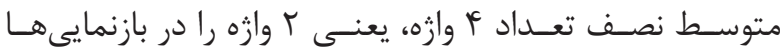

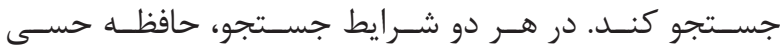

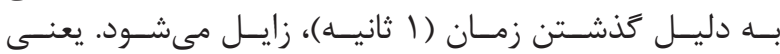

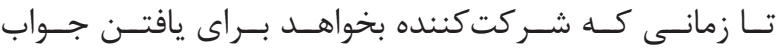

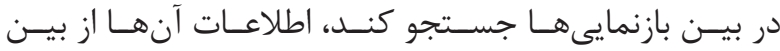

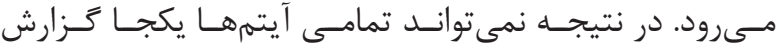

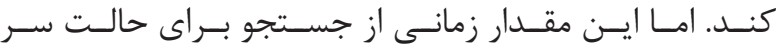

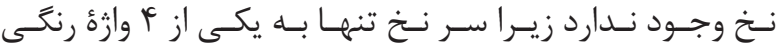

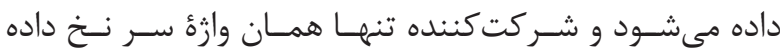

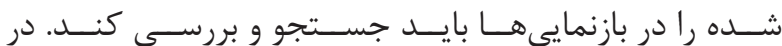

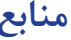

9. Koch C, Tsuchiya N. Attention and consciousness: related yet different. Trends in cognitive sciences. 2012; 16(2): 103-5.

10. Tsuchiya N, Koch C. The relationship between consciousness and top-down attention. The Neurology of Conciousness: Elsevier; 2016. p. 71-91.f

11. Van Boxtel JJ, Tsuchiya N, Koch C. Consciousness and attention: on sufficiency and necessity. Frontiers in Psychology. 2010; 1: 217.

12. Lamme VA. How neuroscience will change our view on consciousness. Cognitive Neuroscience. 2010; 1(3): 204-20.

13. Clarke J, Mack A. Iconic memory for the gist of natural scenes. Consciousness and cognition. 2014; 30: 256-65.

14. Sperling G. The information available in brief visual presentations. Psychological monographs: General and applied. 1960; 74(11): 1.

15. BlockN.Perceptual consciousness overflows cognitive access. Trends in cognitive sciences. 2011;15(12):567-75.

16. Mack A, Erol M, Clarke J. Iconic memory 
is not a case of attention-free awareness.

Consciousness and cognition. 2015; 33: 291-9.

17.MackA,ErolM,ClarkeJ. Whenexpectationconfounds iconic memory: A reply to Bachmann and Aru. 2017.

18. Mack A, Clarke J, Erol M. Attention, expectation and iconicmemory:AreplytoAruandBachmann(2017).2018.

19. Mack A, Clarke J, Erol M. Reply to Bachmann and Aru. Consciousness and cognition. 2015; 35: 156-7.

20. Aru J, Bachmann T. Expectation creates something out of nothing: The role of attention in iconic memory reconsidered. Consciousness and cognition. 2017; 53: 203-10.

21. Bachmann T, Aru J. Comments on how Mack et al. see iconic memory. 2015.

22. MacLeod CM. Half a century of research on the Stroop effect: an integrative review. Psychological bulletin. 1991; 109(2): 163.

23. MacLeod CM. The Stroop task: The" gold standard" of attentional measures. Journal of Experimental Psychology: General. 1992; 121(1): 12. 